\title{
Measurement of the Transverse Polarization of Electrons Emitted in Free Neutron Decay
}

\author{
Adam Kozela*, Aleksandra Białek \\ Instytut Fizyki Jadrowej PAN \\ E-mail: adam.kozela@ifj.edu.pl \\ Kazimierz Bodek, Stanisław Kistryn, Marcin Kuźniak, Jacek Zejma \\ Institut Fizyki, Universytet Jagielloński
}

\section{Klaus Kirch}

Paul Scherrer Institut

\section{Gilles Ban, Pierre Gorel, Oscar Naviliat-Cuncic}

LPC-Caen, ENSICAEN, Université de Caen Basse-Normandie, CNRS/IN2P3-ENSI

\section{Nathal Severijns}

Instituut voor Kern- en Stralingsfysica, Katholieke Universiteit Leuven

\section{Elżbieta Stephan}

Institut Fizyki, Universytet Ślaski

\begin{abstract}
Final values of the transverse polarization components $\left(\sigma_{T_{1}}, \sigma_{T_{2}}\right)$ of electrons emitted in the $\beta$ decay of polarized, free neutrons have been determined for the data set comprising events with full goemetrical information taken in nTRV experiment. The T-odd, P-odd correlation coefficient associated with polarization component perpendicular to the neutron polarization and electron momentum, $\sigma_{T_{2}}$, was found to be $R=0.008 \pm 0.015 \pm 0.005$. This value is consistent with time reversal invariance, and significantly improves limits on the relative strength of imaginary scalar couplings in the weak interaction. The value obtained for the correlation coefficient conected with $\sigma_{T_{1}}, N=0.056 \pm 0.011 \pm 0.005$, agrees with the Standard Model expectation, providing an important sensitivity test of the experimental setup.
\end{abstract}

European Physical Society Europhysics Conference on High Energy Physics

July 16-22, 2009

Cracow, Poland

\footnotetext{
${ }^{*}$ Speaker.
} 
One of the most important problems of the standard model (SM) is connected with the incomplete knowledge of physics of the combined charge and parity symmetry violation (CPV), or via CPT theorem equivalent, time reversal symmetry violation (TRV). The Kobayashi-Maskawa mechanism responsible for CPV in the current formulation of the SM accounts for the CPV discovered so far in kaon and B-meson systems, but it turns out to be too weak to explain such a basic observation like the matter-antimatter asymmetry of the present Universe. It can be shown that even tiny contributions of exotic couplings can account for the required level of CPV. In this context, observation of new CP- or T-violating phenomena is very important, especially in systems built of light quarks only, where the contribution of Kobayashi-Maskawa mechanism is vanishingly small. This applies to all nuclear beta decays, but due to its simplicity, free neutron decay is particularly interesting. Unit charges involved and relatively small decay asymmetry parameter (A) result in small final state interaction corrections which, in absence of the nuclear and atomic structure, can be calculated with a very good precision. This is specially important since the final state interaction, as the second order effect, can mimic TRV.

The decay rate distribution from polarized neutrons is proportional to [1]:

$$
W(\mathbf{J}, \hat{\sigma}, E, \mathbf{p}) \propto 1+\frac{\mathbf{J}}{J} \cdot\left(A \frac{\mathbf{p}}{E}+R \frac{\mathbf{p} \times \hat{\sigma}}{E}+N \hat{\sigma}\right) \ldots
$$

where $\mathbf{J}$ is the neutron spin, $\hat{\sigma}$ is a unit vector onto which the electron spin is projected, $E, \mathbf{p}$ represent energy and momentum of the emitted electron and $A$ is beta decay asymmetry parameter. $N$ and $R$ are the correlation coefficients which, for neutron decay with the SM assumptions $C_{V}=$ $C_{V}^{\prime}=1, C_{A}=C_{A}^{\prime}=\lambda=-1.27$, and allowing for a small admixture of the scalar and tensor couplings $C_{S}, C_{T}, C_{S}^{\prime}, C_{T}^{\prime}$, can be expressed as:

$$
\begin{aligned}
& N=-0.218 \cdot \operatorname{Re}\left(\left(C_{S}+C_{S}^{\prime}\right) / C_{V}\right)+0.335 \cdot \operatorname{Re}\left(\left(C_{T}+C_{T}^{\prime}\right) / C_{A}\right)-\frac{m}{E} \cdot A, \\
& R=-0.218 \cdot \operatorname{Im}\left(\left(C_{S}+C_{S}^{\prime}\right) / C_{V}\right)+0.335 \cdot \operatorname{Im}\left(\left(C_{T}+C_{T}^{\prime}\right) / C_{A}\right)-\frac{m}{137 p} \cdot A,
\end{aligned}
$$

where $m$ is the electron mass. Only taking into account final state interactions (last terms in Eqs 2 and 3) $R$ and $N$ become different from zero. However, since $R_{F S I} \approx 0.0006$ is below the sensitivity of the present experiment, any larger absolute value measured would provide a hint for the existence of exotic couplings, and in consequence a new source of TRV. The value of $N_{F S I} \approx 0.068$ is well within the reach of this experiment. Its determination provides an important sensitivity test of the experimental apparatus.

The experiment was performed at the FUNSPIN beam line at the cold neutron source SINQ, Paul Scherrer Institute, Villigen, Switzerland. The Mott polarimeter [2] consists of two identical modules, arranged symmetrically on both sides of vertically polarized cold neutron beam (Fig. 1). Each module contains a multi-wire proportional chamber (MWPC) for electron tracking, a Mott foil (1-2 $\mu \mathrm{m} \mathrm{Pb})$ for electron polarization analysis, and a scintillator hodoscope for the electron total energy measurement. Two kinds of events were recorded in the experiment: single track events (only one reconstructed track segment on the hit scintillator side, used for a precise neutron beam polarization measurement) and V-track candidates: events with two reconstructed segments on one side and one segment accompanied by a scintillator hit on the opposite side (red in Fig. 1). Using the V-track rate asymmetry for opposite beam polarization states as a function of the angle between 


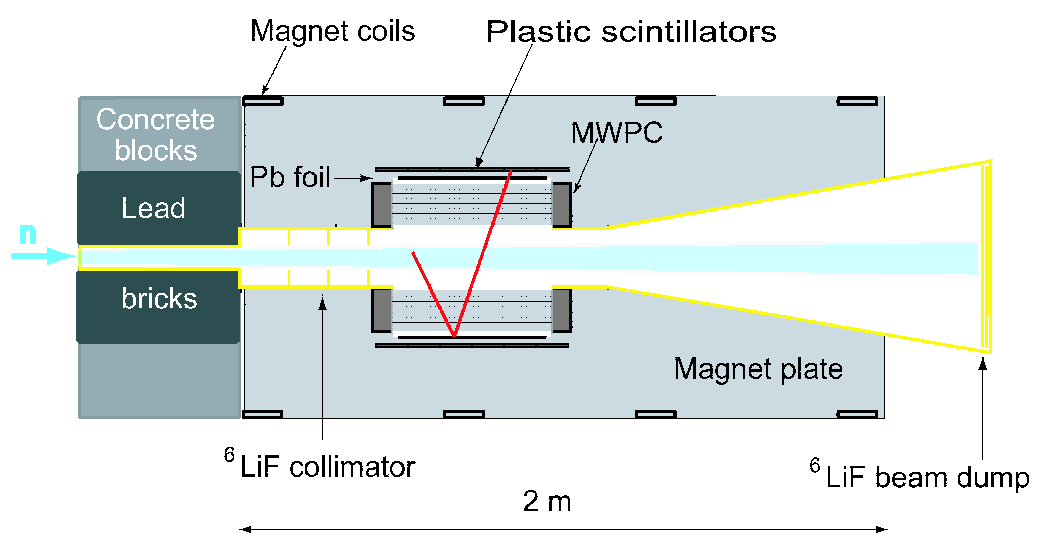

Figure 1: Schematic top view of the experimental setup.

the decay plane (defined by neutron polarization and electron emission momentum) and the scattering plane (defined by electron momenta before and after scattering) this apparatus allows for the simultaneous measurement of both transverse components of the electron polarization, providing access to both the real and imaginary parts of the scalar and tensor couplings.

The reported result [3] comprises independent analysis of events with full geometrical information taken in four data collection periods. Specified periods feature different basic conditions like beam polarization, Mott foil thickness and acquired statistics (see Table 1). However, the real brake-through achieved between 2004 and 2006 followed from substantial improvements of the detector performance and event trigger electronics.

Table 1: Summary of results obtained in all data collection periods. Statistical and systematic uncertainties follow the experimental values. $n$ represents the total, background corrected number of Mott-scattered events, $P$ is the average neutron beam polarization and $N_{S M}$ is the SM value of the $N$ coefficient. Results of two independent $N$ extraction procedures are given [3].

\begin{tabular}{ccccccc} 
Run & $n$ & $P \times 10^{2}$ & $N_{S M} \times 10^{3}$ & $N_{s r} \times 10^{3}$ & $N \times 10^{3}$ & $R \times 10^{3}$ \\
\hline 2003 & 19000 & $80.3 \pm 1.3 \pm 1.6$ & $71 \pm 1$ & $110 \pm 108 \pm 27$ & $82 \pm 97 \pm 31$ & $-89 \pm 143 \pm 38$ \\
2004 & 74000 & $44.2 \pm 0.4 \pm 1.5$ & $68 \pm 1$ & $144 \pm 92 \pm 15$ & $70 \pm 86 \pm 17$ & $-117 \pm 140 \pm 26$ \\
2006 & 312000 & $80.0 \pm 1.0 \pm 1.5$ & $68 \pm 1$ & $79 \pm 32 \pm 7$ & $86 \pm 30 \pm 8$ & $-11 \pm 42 \pm 9$ \\
2007 & 1747000 & $77.4 \pm 0.2 \pm 0.7$ & $68 \pm 1$ & $54 \pm 12 \pm 5$ & $51 \pm 12 \pm 6$ & $12 \pm 16 \pm 6$ \\
\hline Total & 2152000 & & & $59 \pm 11 \pm 4$ & $56 \pm 11 \pm 5$ & $8 \pm 15 \pm 5$
\end{tabular}

This is the first determination of the $R$ correlation coefficient in neutron beta decay, while the $N$ coefficient has not been ever addressed experimentally before. In Fig. 2 the obtained results have been plotted onto exclusion plots containing all experimental information available from neutron and nuclear beta decays [4]. Shown are normalized scalar and tensor coupling constants $S=\left(C_{S}+\right.$ $\left.C_{S}^{\prime}\right) / C_{V}$ and $T=\left(C_{T}+C_{T}^{\prime}\right) / C_{A}$. Although the present accuracy of the determination of the $N$ coefficient does not improve the already strong constraints on the real part of the couplings (left panel), the obtained result is consistent with the existing data and, in addition, adds confidence to the validity of the extraction of $R$. The new experimental value of the $R$ correlation coefficient 
significantly constrains scalar couplings beyond the limits from all previous measurements (right panel). The result is consistent with the SM and the Time Reversal Invariance.
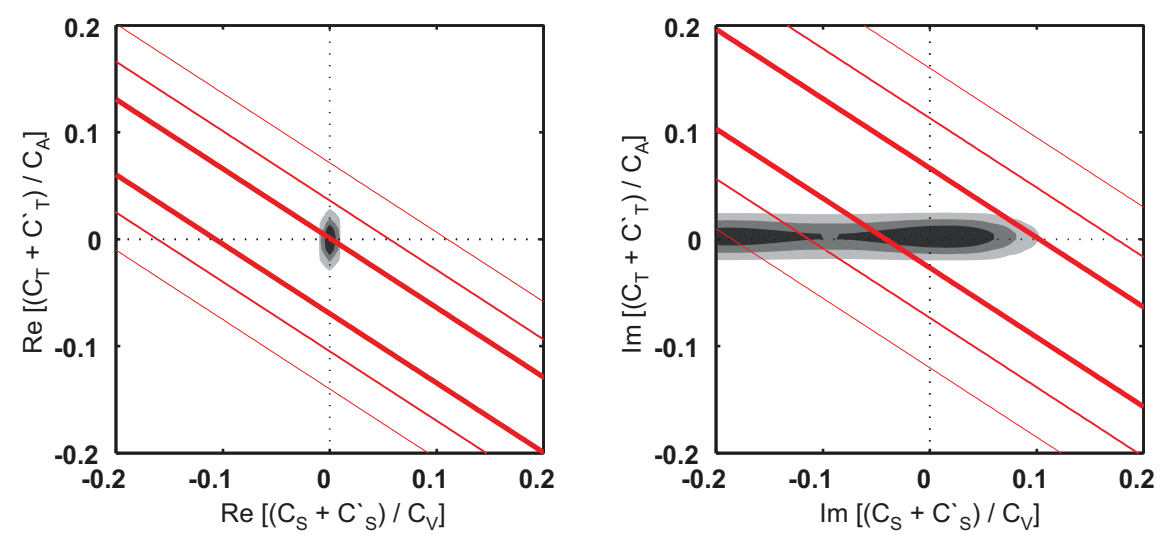

Figure 2: Experimental bounds on the scalar vs. tensor normalized couplings. The grey areas represent the information available before, while the lines represent the limits resulting from the present experiment [3]. 1-, 2- and 3- sigma confidence levels are indicated by decreasing line thickness and intensity of grey areas.

No further data taking is planned with the detector setup presented above. However, some improvement of the final limits can still be achieved by analysis of an event class neglected so far. As shown by the preliminary analysis, especially interesting are the events with scattering vertex reduced to only one segment in horizontal projection, compliant with electrons scattered almost within the vertical plane. The slightly inferior quality of such events, caused by the lack of important matching parameters, is fully compensated by their higher average sensitivity to the $R$ correlation coefficient. Several additional factors enhancing the significance of those events (horizontal beam location, better positional resolution of MWPC in vertical projection and much lower double track resolution in horizontal direction) have opposite influence on events scattered close to the horizontal plane, thus reducing the expectation for a possible improvement of the $N$ correlation coefficient.

This work was supported by the Polish Committee for Scientific Research under the Grant No. 2P03B11122 and by an Integrated Action Program Polonium (Contract No. 05843UJ). Part of the computation work was performed at ACK Cyfronet, Kraków. The collaboration is grateful to PSI for excellent support and kind hospitality.

\section{References}

[1] J. D. Jackson, S. B. Treiman and H. W. Wyld, Nucl. Phys., 4, 206 (1957).

[2] G. Ban at al., Nucl. Instrum. Methods Phys. Res., Sect. A 565, 711 (2006).

[3] A. Kozela at al., Phys. Rev. Lett. 102, 172301 (2009).

[4] N. Severijns, M. Beck and O. Naviliat-Cuncic, Rev. Mod. Phys. A 78, 991 (2006). 